\title{
Utilizing computer-based resources back home when conducting research and teaching overseas
}

\author{
JAMES M. KIELEY \\ Pitzer College, Claremont, California
}

\begin{abstract}
This paper presents information that will help psychologists and other individuals who are planning to conduct research or teach abroad meet their computing-related needs. Factors are discussed that may affect access to network-based resources. An introduction is also provided to newer information technologies, both established and emerging, that may enhance teaching and research efforts conducted in distant settings. The focus is on interactive technologies, such as groupware and computer conferencing, that involve information sharing in real time.
\end{abstract}

The prospect of packing up and moving to a foreign location for an extended period of time may have particular implications for educators and researchers who depend heavily on computer technology to get their work done. In this paper, I attempt to explore technologies, either currently available or on the near horizon, that can lead to wider information access as well as more immediate and direct sharing of information between laboratories and classrooms located continents apart.

A primary motivation for collecting much of the information presented here is the fact that I am relocating for a period of 2 years to a new college that is being established in a relatively isolated area in Japan. I find the idea of moving to another country, without the guarantee of access to the information processing tools upon which I have become progressively more dependent, disconcerting. Among these tools are the actual computers, peripherals, and software to which I have grown accustomed, as well as a number of Internet-based network resources that I have begun to use daily. Some of the information presented here may help others to prepare in advance, so that they will be able to maximize their prospects of maintaining access while they are abroad to many of the computer-based resources that they use now in the United States. Other currently available technologies that may not be widely used now, but that could be of particular benefit while one is working in a foreign location, will be discussed.

In addition to my interest in maintaining access to what I already have available, I am looking at this foreign stay as a chance to do something exciting and new with instructional technology. Emerging technologies may go a long way toward minimizing the limitations that physical distance places on the sharing of information across laboratories and classrooms. Two of these technologies, groupware and computer conferencing, will be briefly discussed and possible applications will be suggested.

Correspondence can be addressed to J. Kieley, Miyazaki International College, 1405 Kano, Kiyatake-cho, Miyazaki-gun, Miyazaki 889-16, Japan.
The common thread in this material is how information technology can be used to minimize the losses and maximize the gains in teaching and conducting research in a foreign setting.

\section{Foreign Network Resources}

Much as personal computers assume a vital role in a modern academic's general professional productivity, many of the remote resources that they allow people to access are becoming ever more important in professional communications. Internet access is becoming so critical that some academics would not consider a foreign assignment where access to the service was unavailable. For many educators, network resources are also beginning to assume a library-like level of importance as part of the extended classroom.

During the past year, the quantity and variety of Internet-accessible resources have continued to grow at an impressive rate, as has public interest. Large numbers of commercial organizations are now using the Internet to supplement business communications and to provide technical support for their products. Significantly, Internet-related databases and software have improved considerably, to the point at which it is now possible to conduct a systematic search for a particular category of information. One indicator of how Internet interest and use has grown is that fact that the number of Internet guides published in book form has approximately tripled over each of the past 2 years (see Figure 1).

Last year, I discussed a number of Internet services and resources that may be of particular value to psychologists (Kieley, 1993). Among these were FTP (which enables remote transfers of virtually any file), Telnet (which permits remote logins to distant computers), and various Internet navigation systems (e.g., Gopher, WAIS, and World-Wide Web).

Table 1 lists a variety of Internet-accessible services that can be of particular value to academics working abroad. First on the list of obviously desirable services would be electronic mail, which is finding ever increasing use for both professional and personal communications. Gateways 
Intemet Books Published 1990 - October 1993

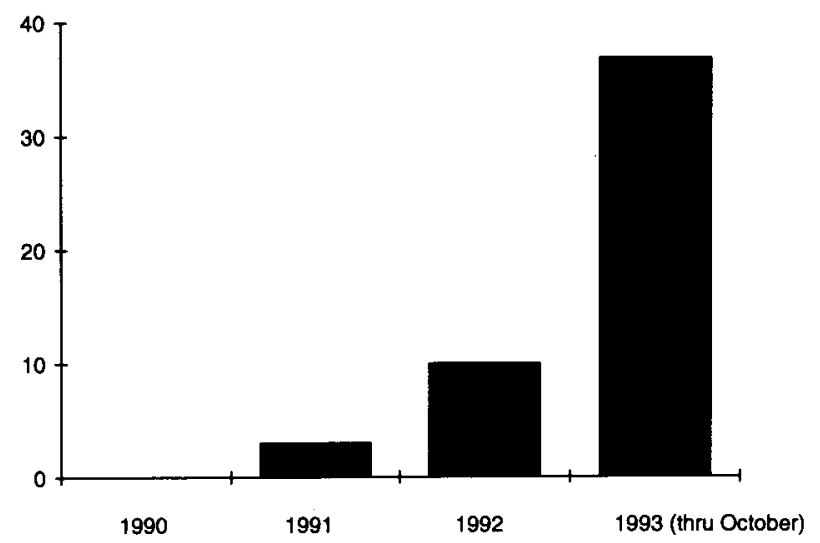

Figure 1. Bar chart representing the number of Internet-related books published since 1990 . The column for 1993 covers through the end of October of that year.

from the Internet to CompuServe and other commonly available dial-up services make e-mail an affordable communication alternative to the telephone that is available in many parts of the world.

Another Internet service that may be of value abroad is FTP, which permits the transfer of files that are either too large for e-mail or that are in a format that some electronic mail programs cannot process. Software that makes FTP transfers very easy and intuitive is now available for most types of personal computers.

Telnet is another Internet service that can be of value to individuals with access to computers located in the United States; it offers software and services that are not available in a foreign location. For example, the computers that I will have access to in Japan will not have the BMDP statistical package available that I use on a regular basis. I plan to use Telnet to connect to a computer in California, which will continue to provide me with BMDP access. I will use FTP to upload the data sets that will be generated in Japan to the computer I will conduct analyses on in California. Telnet is also a valuable tool for accessing on-line holdings and indexes of many distant libraries and database services. In a non-English speaking country like Japan, the limited access to many English language journals will likely be frustrating at times. The ability to locate materials that may be electronically retrievable through on-line database services such as CARL or Knowledge Index, or that may be available through interlibrary loan programs, will be important.

TALK is a complement to e-mail that is available to many academics who generally neglect to take advantage of it. Essentially, it allows two people logged in at the same time in different locations to exchange typed messages in real time. Although TALK may not be as spontaneous or convenient as a costly transcontinental telephone call, it is more immediate and interactive than an e-mail message.
USENET news was originally designed as a forum for permitting individuals located all over the world to share information on a wide variety of topics. USENET may be useful in a foreign country both to keep up on some subject of topical interest and as a cost-effective way to obtain technical help from one of the numerous computerrelated discussion forums that are available. USENET technology can also be used to provide other types of information services. The K12 USENET service can be made available to academics living abroad to support the education of their school-age children (Murray, 1993). A commercial USENET service called Clarinet provides an up-to-date electronic news feed that is distributed in several hundred hierarchically organized categories of English language articles in a format resembling a daily newspaper.

Internet navigational servers located all over the world running software such as Gopher, WAIS, and WorldWide Web can be accessed from a personal computer attached to the Internet. The range of services available through these servers is too vast to describe here. The interested reader is referred to any of several books on using the Internet, including those by Krol and Loukides (1992) and Dern (1993). However, the only real way to appreciate the variety of information available through Internet navigators is to take a hands-on approach. A particularly appealing aspect of this technology is the fact that a local information server can be customized by setting up links to selected information available from other servers all over the world. This will allow us to set up a server in Japan that will provide easy access to information that is of particular local interest, without inundating people with other information that may have less local appeal.

Table 1

Various Internet Services of Use to Academics Working Abroad

\begin{tabular}{|c|c|c|}
\hline Service & Traffic & Minimal Connection \\
\hline E-mail & low & dial-up service \\
\hline Talk & low & direct (any type) \\
\hline FTP & low & direct (any type) \\
\hline Telnet & low & direct (any type) \\
\hline USENET & moderate & direct (any type) \\
\hline
\end{tabular}

Technical support

Clarinet

$\mathrm{K} 12 \mathrm{Net}$

Navigation Servers moderate-high

Gopher direct (any type)

World-Wide Web (WWW)* direct $(\geq 56 \mathrm{~Kb})$

WAIS direct (any type)

$\mathrm{A} / \mathrm{V}$ conferencing high direct (ISDN or $\mathrm{Tl}$ )

Note-Contained in the list is an estimation of the network traffic typically associated with a given service as well as an indication of the type of connection that is minimally needed to support it. Dial-up service (e.g., CompuServe) connection speeds: $1,200-14,400$ bps. Direct Internet (UUCP or TCP/IP) connection speeds: SLIP and PPP (14.4Kbps), Leased line (56-64Kbps), ISDN (128Kbps), Fractional Tl ( >64Kbps), T1 (1.544Mbps), T3 (45Mbps). *WWW requires higher connection speeds to work with certain client software and for reasonable performance with data intensive services such as accessing audio and video archives. 
Internet-accessible services can vary in many ways. As is outlined in Table 1, these services can differ a great deal in the amount of network traffic that they generate. The amount of traffic is a function of the amount of activity at each end of the connection (i.e., how much information is being sent back and forth), which to a large extent is determined by the form of the information (whether it is text, graphics, audio, video, or some combination). The amount of traffic affects the minimal speed or bandwidth of the connection that is necessary to support the service. Network traffic is additive, so if there are several connections that are all attempting to send information over the same cable at the same time, the minimal bandwidth recommended for a particular service listed in Table 1 will be inadequate if reasonable performance is expected.

Although higher education sites in many parts of the world have been connected to the Internet for some time, the likelihood that a given school located abroad has Internet access is less than it is in the United States. If Internet connectivity does exist, the range and quality of services available will be affected by the speed of the local connection to the nearest regional network backbone. Internet connections between foreign institutions are likely to be slower than those between comparably sized United States colleges and universities. Even when this is not the case, it is important to realize that when one is trying to use a bandwidth-intensive application, the maximum rate of information exchange will be limited (or a bottleneck will exist) at the point where the capacity is the lowest. Therefore, it may be useful to contact a colleague or the computing department at a prospective foreign institution in advance in order to discuss the sorts of specific network needs that one might have. This may help one to avoid disappointments. The same people may be invaluable in helping one decide what, if any, computing equipment one should plan to bring along and how it should be configured.

\section{New Technologies for Research and Instruction}

Two relatively new technologies that may soon have a great impact on academics working abroad are groupware and network-based computer conferencing. Some would argue that computer conferencing should most accurately be thought of as a subset of groupware, in that groupware can broadly be defined as "an application that allows groups of individuals to work on common projects in a shared environment" (Briere, 1991). For the purposes of this discussion, I will more narrowly define groupware as software that permits multiple networked computer users to access and update simultaneously the same database or document.

Videoconferencing is a technology that is already available through a variety of means, including satellite transmissions and dedicated fiber optic phone lines. The term computer conferencing is used here to underscore that this discussion will focus on interactive real-time audio and video transmissions that can be achieved over wide-area computer networks such as the Internet. Computer conferencing can also support storyboarding, or the ability to present and modify graphical images that are simultaneously visible on two or more computer screens at each end of a network link.

\section{Groupware}

Groupware by design is intended to follow a clientserver model of information exchange. A dedicated or a nondedicated machine can act as the server dispersing information to multiple client computers. The client computers can theoretically be different types of machines running different types of software to support the connection to the server. Commercial versions of integrated groupware systems are now available, including the wellpublicized Lotus Notes (Stevenson, 1993). Also available to support the groupware approach is computer programming technology such as NeXT Computer's distributed objects, which permit the rapid development of programs that can support the sharing of information over TCP/IP (a network protocol) networks such as the Internet.

An application of groupware technology of interest to me is to have software available that will allow individuals located on two continents to update dynamically a shared repository of experimental information. I will be collecting data from subjects living in Japan while comparative data is collected from subjects residing in California. In order to monitor the progress of the research accurately, I will be interested in having immediate access to an up-to-date cumulative record of both the subject information and the actual experimental data that will be collected. Although it would certainly be possible for the two laboratories to maintain separate databases that would be periodically manually updated and merged, the logistics of taking this approach can become confusing and mistakes can easily be made.

Other useful applications of groupware exist for both research-related and teaching purposes. One possible use would consist of collaboration on manuscripts or editing projects. There are obvious problems that can be imagined if two people were trying to work on the same document at the same time without understanding what one another was doing. The idea of text disappearing and being replaced before one's eyes without explanation could be quite maddening, but the idea of combining real-time document sharing with the ability to annotate or discuss changes with an accompanying two-way audio channel carried over the same network is another matter. This type of technology has real promise, particularly for individuals living at great distances apart who are collaborating on long-term projects. It is currently being used with success in research and business settings (Brittan, 1992).

Groupware is also finding its way today into education, particularly in elementary and high schools where it is used to teach group collaboration skills that will hopefully later transfer into the workplace. Some of the cur- 
rently available software creates a collaborative writing environment that allows students to interact with each other and with their teachers over a network in real time.

Certainly applications for groupware in the college classroom are possible. A simple demonstration of groupware technology that is bundled with the NeXTstep 3.1 development system is called RemoteVote.app. This program presents a simple graphical user interface that allows class attendees to rate an oral presentation on several dimensions. As a user adjusts slider bars representing each dimension, the screen outputs both his/her rating and an average for everyone else on the network who is making an evaluation at that time. This program could certainly be used to provide a unique demonstration of conformity in a social psychology classroom. A simple modification of the program could give accurate feedback (i.e., class averages), random feedback, or no feedback to different groups of students, demonstrating how real or perceived group influence affects individual judgments.

\section{Computer Conferencing}

Internationally accessible multimedia resources are a reality that is already here. Hypertext-based client software for the World-Wide Web Internet navigation servers such as Mosaic (which is available for Macintosh, Windows, and XWindows operating systems) support access to advanced graphics, sound, and video databases located throughout the world, although this multimedia information must be accessed from a hard disk, CD-ROM drive, or similar type of storage device. Computer conferencing is a more resource-intensive multimedia technology that involves the transfer of similar types of information in real time.

As was suggested before, computer conferencing storyboard applications are available that allow multiple users at distant locations to view and work on the same document. There is also the ability to transmit audio comments which may or may not be accompanied by video projections. Although the day may not be here yet when anyone can routinely sit at a desk or terminal and have instant real-time audio-video communications with anyone anywhere in the world at an affordable price, technology is clearly moving in that direction. In preparation for an anticipated video communications boom, several countries are investing enormous amounts of money to develop fiber-optic-based communication links that will eventually extend from every business and school into every home.

Computer conferencing is still at a largely experimental stage. Although the technology works, the full demands that it will eventually place on supporting technologies, as well as its potential impact on society, are not clearly understood. The amount of traffic that can be generated by large numbers of audio-video sessions attempting to travel over the same computer network at the same time will drive engineers to design faster equipment and produce better compression algorithms. This will help ensure that performance will keep up with demand. The abil- ity to conduct business interactively in electronically generated multimedia virtual worlds will certainly affect travel and work habits.

The list of conceivable research and instructional applications for this technology is almost limitless. For example, the possibilities of having computer conferences between collaborating researchers or electronically linking laboratories at great distances would be invaluable. Computer conferencing can potentially open up the world to students and allow them to interact with people all over the planet. The theme of multicultural awareness that is being stressed in education today certainly could benefit from a technology that would lower physical barriers and promote international awareness on a one-on-one basis. Satellite-based and dedicated leased-line videoconferencing is already being used regularly in education (Graham, 1992). Providing similar capabilities over computer networks that are less expensive to operate will make the technology available to a much larger audience.

Recent technological advances have produced compression algorithms that greatly reduce the amount of data that must be transmitted over computer networks to support video. This means that better quality video can be provided at lower cost, since slower and less expensive transmission lines can support low-resolution video transmissions at least.

Although high-quality transmissions require powerful workstations and fast peripheral equipment, limited video transmissions are now possible with common personal computers. One recently announced product for Macintosh and IBM-compatible personal computers called Connect 918 and marketed by NUTS Technologies supports audio and full-motion color computer conferencing as well as screen sharing. The video image is not full screen $(176 \times 144)$, but the size can be doubled by cutting the frame speed in half from 30 frames per second (fps) to 15 fps. Video seen at 15 fps is quite choppy, but it is acceptable for certain applications. This system can be run over local area networks or over analog or digital leased phone lines. The list prices for various system configurations, which include a camera, a hardware card, cables, and software, range from $\$ 4,250$ to $\$ 5,850$. The price does not include the computer, and two such systems would be necessary for any implementation. A higher end program that supports audio, video, application sharing, and storyboarding is ShowMe 2.0. This system is designed to run on SUN workstations connected to TCP/IP networks. One option includes a video board, a camera, and software for $\$ 3,270$.

The performance of these products over a network will largely be determined by the speed of the computer hardware and the speed of the network connection. The higher the quality and the more data-intensive the transmission, the faster the transport medium must be. Reasonable costeffective performance can be obtained over dedicated ISDN (Integrated Services Digital Network) leased lines that have a $128 \mathrm{Kbps}$ bandwidth. Although ISDN capability is not found everywhere, it is commonly available in 
Europe and will be available in most major United States cities by the end of 1994 (Landwehr, 1992). Even better performance can be achieved over T1 network links.

Real-time computer conferencing over the Internet has been going on for well over a year on an experimental basis. One of the reasons why the idea of providing computer conferencing over the Internet is appealing is that there are no usage-specific charges beyond the monthly costs of maintaining a connection.

The most prominent implementation of computer conferencing technology currently seen on the Internet is the M-BONE (Multi-cast Backbone). The M-BONE is an experimental network that runs on top of the Internet and allows the transmission of live audio and video to participating sites. This technology differs from a peer-topeer technology in that any transmission takes place over a shared link and can theoretically be received by many sites. This can be thought of as analogous to a cable television network in which any subscribing site can pick up a transmission. What is unique about the M-BONE is that unlike cable TV it is not privately owned, and it is international in scope. This means that it is possible for educational institutions to send real-time audio and video multicasts to other sites around the world without a specific charge for the service on either the transmitting or the receiving end. Although this technologically may not be immediately applicable in a broad educational context, it does suggest that global Internet-like data networks may be useful for computer conferencing applications.

One practical demonstration of this technology was the Global Schoolhouse project, in which school children in California, Tennessee, and England participated in a video conference during which they discussed water pollution (MacLeod, 1993). Another practical implementation of this technology is the weekly Internet Talk Radio series that features live interviews with various individuals active in network communications; these are multicast over the Internet to sites throughout the world (Ziegler, 1993).

When computer conferencing technology will become an easily accessible common Internet tool has not yet been clearly established. The speed of the United States Internet backbone will be upgraded in coming years in anticipation of increasing traffic. Presumably, the capacity at colleges and universities in the United States and in other countries will continue to grow as well. It is already possible to access many resources over the the Internet that were unthinkable a decade ago. Long-distance highquality computer conferencing may be commonplace 10 years from now. In the interim, expect a continued stream of new tools that will make this a progressively higher quality and more affordable long-distance extension of the laboratory and the classroom.

\section{Conclusion}

Obvious professional and personal rewards can be obtained by spending time teaching and conducting research in a completely different setting from what one is used to. These advantages can easily be offset by disadvantages if one is not able to maintain access to the tools needed to maintain contacts with people and services back home. Existing computer technology can go a long way in affordably bridging information gaps brought about by working in a distant location. Emerging technologies that are promised for the future will go much farther.

\section{REFERENCES}

Briere, D. (1991, September 16). Groupware: A spectrum of productivity boosters. Network World, p. 1.

Brittan, D. (1992). Being there: The promise of multimedia communications. Technological Review, 95, 42-50.

DERN, D. (1993). The Internet guide for new users. New York: McGraw-Hill.

Graham, E. (1992, May 18). Classrooms without walls: Advances in telecommunications promise to transform both learning and teaching. Wall Street Journal, p. R8.

KIELEY, J. M. (1993). Integrating remotely accessible information resources into psychology instruction and research. Behavior Research Methods, Instruments, \& Computers, 25, 287-294.

KROL, E., \& LoukIDEs, M. (1992). The whole Internet user's guide \& catalog. Sebastopol, CA: O'Reilly \& Associates.

LANDWEHR, J. (1992). The golden splice: Beginning a global digital phone network. Unpublished manuscript. Evanston, IL: Northwestern University.

MacLeod, D. (1993, May 6). So near, yet so far apart. Independent, p. 19.

MURRAY, J. (1993). K12 Network: Global education through telecommunications. Communications of the $A C M, 36,36-41$.

Stevenson, T. (1993, June 15). Groupware: Are we ready? PC Magazine, p. 267.

ZIEGLER, B. (1993, August 9). Tune in next time for another "Geek of the Week." Business Week, p. 72. 